\title{
Paidéia: Cadernos de Psicologia e Educação: Relatório de Gestão 2007
}

\author{
Manoel Antônio dos Santos \\ Lucy Leal Melo-Silva \\ Eduardo Name Risk \\ Universidade de São Paulo, Ribeirão Preto-SP, Brasil
}

O presente relatório tem por objetivo apresentar a gestão editorial da revista Paidéia: Cadernos de Psicologia e Educação no ano de 2007, a fim de tornar público seu processo editorial, o que permite orientar e dirimir dúvidas de autores, consultores e leitores, na medida em que esclarece minuciosamente os passos de tramitação dos manuscritos, os critérios de arbitragem, a abrangência do corpo de consultores ad hoc, o tempo, em geral, que decorre para um trabalho ser publicado, a origem institucional/geográfica dos autores que publicaram na revista, além das categorias dos artigos que foram publicados no período considerado. A partir desses dados, é possível que o corpo editorial, consultores ad hoc e, especialmente, os autores possam conhecer de modo sistemático o perfil desta revista e situá-la em relação aos outros periódicos da área, o que contribui para análise da produção editorial atualmente.

Este trabalho está subdividido nas seguintes seções: Espaço editorial, Critérios de arbitragem dos manuscritos submetidos, Processo editorial no ano 2007 e Considerações finais.

\section{Espaço editorial}

A revista Paidéia: Cadernos de Psicologia e Educação é vinculada ao Programa de Pósgraduação em Psicologia da Faculdade de Filosofia, Ciências e Letras de Ribeirão Preto da Universidade de São Paulo - FFCLRP-USP. Tem periodicidade quadrimestral e publica trabalhos originais relacionados à Psicologia, Educação e áreas afins. Seu mandado inicial voltava-se para a área da Educação, passando a contemplar o âmbito da Psicologia e áreas afins a partir de 1996.
Em seus 17 anos de existência, a revista manteve-se diversificada e abrangente, recebendo artigos de diferentes estados do Brasil e de outros países, fruto do intercâmbio sistemático da FFCLRP-USP com centros, laboratórios e grupos de pesquisa do Brasil e de países com os quais o Programa de Pós-graduação em Psicologia do Departamento de Psicologia e Educação vem estruturando e mantendo contato.

Desde 1998, a revista tem sido avaliada pelos critérios Qualis da CAPES (Coordenação de Aperfeiçoamento de Pessoal de Nível Superior) e ANPEPP (Associação Nacional de Pesquisa e Pósgraduação em Psicologia), mantendo nas últimas classificações o conceito A-Nacional, conforme os critérios de normalização, publicação, circulação, autoria, conteúdo e gestão editorial. Cabe salientar que, em 2008, durante o XII Simpósio da ANPEPP, foram discutidos os novos critérios de avaliação Qualis, o que exigirá algumas modificações na estrutura editorial com vistas a atendê-los.

A revista já publicou 39 números, cujos artigos se subdividem em duas ou três perspectivas, conforme o fascículo: a) Pesquisas Teóricas, trabalhos fundamentados em discussões teóricas ou em análises de revisão da literatura, investigações acerca de construtos teóricos que promovem o questionamento de modelos existentes, além de problemas de ordem ética; b) Pesquisas Empíricas, investigações baseadas em dados empíricos pautadas no método científico; c) Relatos de experiência profissional, descrição de procedimentos de intervenção, avaliação de sua eficácia e análise de suas implicações conceituais; d) Seção Especial, ocasionalmente publicada, na qual reúnem-se artigos que discutem temas candentes à Psicologia, Educação e áreas afins, e) Resenhas, que tratam de obras recém-publicadas. 
Ao final de cada número constam informações sobre futuros congressos e simpósios das áreas de Psicologia, Educação e Ciências afins, além das normas de publicação, disponíveis em três idiomas: português, inglês e espanhol. O último fascículo de cada volume contempla a lista dos assessores ad hoc que revisaram manuscritos durante o ano vigente.

A distribuição da revista é gratuita para as bibliotecas de universidades públicas brasileiras vinculadas a cursos de graduação em Psicologia e/ ou Educação (Tabela 1), incluindo-se algumas do exterior, tais como Portugal, Espanha, México e EUA. Isto se traduz, na prática, por um envio de 160 exemplares para universidades e faculdades de

\section{Tabela 1}

Quantidade de fasciculos da revista Paidéia distribuidos conforme o estado de origem das bibliotecas brasileiras e Programas de Pósgraduação nas áreas de Psicologia e Educação

\begin{tabular}{lc}
\hline \multicolumn{1}{c}{ Estado } & $\begin{array}{c}\text { Número de } \\
\text { fascículos } \\
\text { distribuídos }\end{array}$ \\
\hline São Paulo & 61 \\
Rio de Janeiro & 12 \\
Paraná & 11 \\
Minas Gerais & 10 \\
Rio Grande do Sul & 10 \\
Santa Catarina & 8 \\
Espírito Santo & 7 \\
Distrito Federal & 4 \\
Bahia & 4 \\
Ceará & 3 \\
Goiás & 3 \\
Mato Grosso & 3 \\
Mato Grosso e Mato Grosso do Sul & 3 \\
Pernambuco & 3 \\
Pará & 2 \\
Piauí & 2 \\
Acre & 1 \\
Alagoas & 1 \\
Amapá & 1 \\
Amazonas & 1 \\
Maranhão & 1 \\
Paraiba & 1 \\
Rio Grande do Norte & 1 \\
\hline
\end{tabular}

diversos estados brasileiros, além de cópias extras voltadas para Programas de Pós-graduação nas áreas de Psicologia e Educação.

A indexação da revista iniciou-se em 2000, junto à Base de Dados LILACS, seguida do INDEXPSI em 2002. Paralelamente, houve seu credenciamento junto ao SIBI (Sistema Integrado de Bibliotecas da USP), órgão que analisa os periódicos vinculados à Universidade de São Paulo e prevê, após aprovação, um auxílio básico para a confecção das revistas. Esse credenciamento permitiu dinamizar a produção dos fascículos e também obter o aval da Universidade para encaminhar sua inclusão no sistema SciELO (Scientific Electronic Library Online).

Além do financiamento via SIBI, a revista tem recebido apoio regular do Conselho Nacional de Desenvolvimento Científico e Tecnológico (CNPq) desde 2003. De 2002 em diante a Paidéia passou a vincular-se à Associação Brasileira de Editores Científicos (ABEC). Entre 2003 e 2004 a Paidéia foi incluída em três novas bases de dados para indexação: PsycINFO (American Psychological AssociationEUA), CLASE (México) e PSICODOC (Espanha). Desde 2003, a revista passou a ser disponibilizada online na página da Faculdade de Filosofia, Ciências e Letras. Um ano e meio depois, foi submetida à avaliação junto ao sistema SciELO, tendo sido aceita em 2006 e disponibilizada em 2007.

Atualmente a revista encontra-se indexada nas seguintes bases:

- SciELO - Scientific Electronic Library Online (FAPESP-Fundação de Amparo à Pesquisa do Estado de São Paulo/BIREMECentro Latino-Americano e do Caribe de Informação em Ciências da Saúde).

- PsycINFO (American Psychological Association)

- LILACS - Literatura Latino-Americana e do Caribe em Ciências da Saúde (Centro Latino-americano y de Caribe de Informaciones em Ciências de la Salud)

- CLASE-Citas - Latinoamericanas en Ciências Sociales y Humanidades (Universidad Nacional Autónoma de México) 
- PSICODOC (Colégio Oficial de Psicólogos de Madrid/Universidad Complutense de Madrid)

- INDEXPSI Periódicos (CFP-Conselho

Federal de Psicologia/PUCCAMP-Pontifícia Universidade Católica de Campinas)

No que tange ao corpo editorial, a revista Paidéia: Cadernos de Psicologia e Educação teve como Editora Responsável, de 1998 a 2007, a Profa. Dra. Zélia Maria Mendes Biasoli-Alves, que infelizmente veio a falecer em julho de 2007, deixando a todos o legado de sua calorosa e apaixonada dedicação a este periódico. Nesse período, a Paidéia se consolidou paulatinamente como espaço editorial aglutinador dos conhecimentos produzidos na área da Psicologia e Educação. Após a morte precoce da Profa. Zélia, o Prof. Dr. Manoel Antônio dos Santos assumiu o cargo de Editor Responsável.

Como editora associada, desde 2002, a revista conta com o apoio da Profa. Dra. Eucia Beatriz Lopes Petean. A partir de 2007, com o novo editor,

\section{Tabela 2}

Abrangencia dos membros do Conselho Editorial da revista Paidéia, conforme fliaçäo institucional e origem geográfica

\begin{tabular}{|c|c|}
\hline Instituição / País & $\%$ \\
\hline $\begin{array}{l}\text { Universidade de Brasilia, Brasil } \\
\text { Universidade Federal do Rio de Janeiro, } \\
\text { Brasil } \\
\text { Universidade de São Paulo, Brasil } \\
\text { Universidade Estadual Paulista, Brasil }\end{array}$ & 33 \\
\hline $\begin{array}{l}\text { Unversidade de Coimbra, Portugal } \\
\text { Universidade de Lisboa, Portugal } \\
\text { Universidade do Porto, Portugal }\end{array}$ & 25 \\
\hline $\begin{array}{l}\text { Université du Québec à Montreal, } \\
\text { Canadá } \\
\text { Université du Québec à Trois Rivières, } \\
\text { Canadá }\end{array}$ & 16 \\
\hline $\begin{array}{l}\text { Universidade Nacional de Colómbia, } \\
\text { Colômbia }\end{array}$ & 8 \\
\hline University of Alabama, EUA & 8 \\
\hline $\begin{array}{l}\text { Institut National de la Sante et la } \\
\text { Recherche Medicale, França }\end{array}$ & 8 \\
\hline
\end{tabular}

o Prof. Dr. Antonio dos Santos Andrade e a Profa. Dra. Lucy Leal Melo-Silva assumiram a função de Editores Associados, ao lado da Profa. Dra. Eucia Beatriz Lopes Petean. Atualmente, a Comissão Editorial é composta pelo Prof. Dr. Geraldo Romanelli, Profa. Dra. Marina Rezende Bazon, Prof. Dr. Marcus Vinicius da Cunha e Prof. Dr. Reinaldo Furlan, todos do Departamento de Psicologia e Educação da FFCLRP-USP. O Conselho Editorial, estruturado em função dos temas abordados nos artigos submetidos, é constituído por nomes expressivos da comunidade acadêmica nacional e internacional. A filiação institucional de seus membros é relatada na Tabela 2.

\section{Critérios de arbitragem dos manuscritos submetidos}

Os trabalhos submetidos à revista devem se enquadrar nas seguintes categorias: (1) relato de pesquisa; (2) estudo teórico; (3) relato de experiência profissional; (4) revisão crítica da literatura; (5) comunicação breve; (6) nota técnica; (7) resenha. A critério do Editor é permitido também a publicação de notícias, fatos ou eventos relacionados à Psicologia e à Educação. A aceitação do manuscrito submetido para avaliação, de acordo com as categorias acima descritas, se dá tendo como pressupostos: que se trata de um trabalho original, ou seja, não foi publicado nem está sendo submetido para publicação em outro periódico, todos os autores aprovaram sua submissão, qualquer pessoa citada como fonte de comunicação pessoal aprovou a citação, além da estrita observância dos critérios éticos na realização de pesquisas.

A tramitação de um manuscrito inicia-se apenas se ele atender às diretrizes de preparação e submissão de trabalhos descritas nas normas, atentando-se para sua padronização formal, além do envio da carta de encaminhamento assinada por todos autores, que deve versar sobre a cessão dos direitos autorais do manuscrito à revista, caso venha a ser publicado, a garantia da observância dos princípios éticos em pesquisa, além da indicação da categoria em que se insere o trabalho. Caso atenda a esses requisitos, o manuscrito será inicialmente apreciado pelo editor, que avaliará sua pertinência à linha temática da revista. Em caso negativo, o fato é imediatamente comunicado aos autores. 
Em caso positivo, o editor poderá valer-se do apoio de membros do corpo editorial para indicar possíveis consultores ad hoc do trabalho. A revista pauta-se no modelo da revisão cega por pares da comunidade científica (peer review-blind review), que por gozarem de legitimado reconhecimento em sua área de pesquisa, supõe-se que estejam habilitados a avaliar os trabalhos a eles confiados. De acordo com este procedimento, tanto a identidade de autores, como de revisores, são mantidas incógnitas, o que pode favorecer julgamentos isentos por parte dos avaliadores.

Cabe ao assistente editorial convidar os assessores indicados pelo editor a revisarem os manuscritos a eles designados, com base na consulta do trabalho na íntegra, do crivo de avaliação e das normas da revista. Caso qualquer consultor, por variados motivos, esteja impedido de avaliar com isenção o manuscrito remetido, deverá dar ciência do fato ao editor, que cancelará o convite, em função do conflito de interesses. Na ausência de tais interposições, cabe ao revisor retornar a avaliação no prazo estabelecido pela secretaria da revista, podendo solicitar extensão desse, caso necessite.

As seguintes diretrizes de avaliação dos manuscritos são adotadas pela revista Paidéia: (a) clareza dos objetivos; (b) correção, sequiência lógica e coerência do conteúdo; (c) adequação da metodologia empregada, frente aos objetivos do trabalho; (d) contribuição para o avanço do conhecimento na área. Pautando-se nestes quesitos, os avaliadores deverão emitir o parecer final, qual seja: (a) aprovação do manuscrito; (b) aprovação mediante modificações; (c) indicação de extensa reformulação, para re-submissão; (d) recusado para publicação. Cada uma destas categorias deve ser justificada em seção à parte do parecer. Os revisores podem também realizar comentários no corpo do manuscrito.

Cada trabalho é avaliado, no mínimo, por dois consultores ad hoc, que emitem pareceres independentes. Recebidas as avaliações, o assistente editorial encaminha-las-á ao editor, que redigirá a Carta do Conselho Editorial referente ao manuscrito original, isto é, uma apreciação ampla do trabalho, baseada no cotejamento dos pareceres e em seus comentários. Nessa carta, em seção à parte, é reproduzida a versão integral dos pareceres dos revisores, sendo finalizada pela nota do manuscrito, indicativa de sua situação, qual seja: aprovação sem reformulações; aprovação mediante reformulações; re-submissão conforme extensa reformulação; recusa do manuscrito. Dificilmente a versão original de um trabalho é aceita plenamente, já que as orientações dos assessores e do editor constituem-se em estratégias para seu aperfeiçoamento. O Editor pode solicitar apoio de membros do Conselho Editorial com vistas à apreciação geral do manuscrito e redação da carta do Conselho Editorial, participando as avaliações encaminhadas pelos revisores, além de compartilhar seus argumentos referentes ao trabalho. Caso os pareceres emitidos pelos revisores sejam discordantes, na medida em que há quem aprove o trabalho mediante modificações ou não da versão original, e quem o recuse, cabe ao Editor solicitar uma terceira avaliação, em geral realizada pelos membros do Conselho Editorial, de modo que as dúvidas sejam dirimidas, e o parecer final possa ser concluído.

A Carta do Conselho Editorial é encaminhada aos autores, que deverão apresentar, em um período de 20 a 30 dias, a primeira versão reformulada do manuscrito, caso o corpo editorial tenha decidido pela continuidade de sua tramitação. Essa versão deve ser acompanhada de carta-resposta endereçada ao Conselho Editorial, devendo conter justificativas das alterações efetuadas no texto original, além de réplica às argumentações dos consultores ad hoc. $\mathrm{O}$ assistente editorial encaminha o texto reformulado, a carta-resposta dos autores, além da carta do Conselho Editorial aos consultores ad hoc que avaliaram o manuscrito original. Cabe a eles apreciarem se os autores acolheram suas sugestões e se justificaram com pertinência aquelas não acatadas. Desta feita, emitirão novo parecer, a saber, se a versão reformulada encontra-se em condições de publicação, ou se ainda prescinde de modificações, podendo também recusar o manuscrito re-submetido.

O Editor novamente coteja os pareceres, redige sua apreciação geral e encaminha a Carta do Conselho Editorial referente à primeira reformulação 
do manuscrito, observando processo semelhante ao decorrido na apreciação da versão original. Caso sejam recomendadas reformulações, os autores deverão apresentar a segunda versão reformulada do manuscrito no prazo de 20 a 30 dias, além da cartaresposta endereçada ao Conselho Editorial, da mesma maneira que procederam na submissão da primeira versão reformulada. Novamente, o editor encaminha o material aos consultores ad hoc, podendo consultar apenas um deles, que emitirão novo parecer. Caso decidam pela aprovação da presente versão do trabalho, caberá ao editor o julgamento final, ou seja: aprová-lo ou não para publicação, podendo valer-se, quando necessário, do apoio dos colegas do corpo editorial. O parecer final do Conselho Editorial será comunicado aos autores. Em caso de aceite, dar-seá seguimento aos procedimentos finais visando à publicação do manuscrito.

A versão final do trabalho é encaminhada para editoração e depois aos bibliotecários da Biblioteca Central da Prefeitura do campus de Ribeirão PretoUniversidade de São Paulo, que realizarão sua padronização de acordo com as diretrizes da quinta versão do Manual de Publicação da American Psychological Association (American Psychological Association [APA], 2001). Posteriormente, o trabalho é revisado pelo assistente editorial e pelo Conselho Editorial, lembrando que o Editor reserva-se o direito de realizar pequenas alterações na redação do texto, desde que não alterem o sentido do que os autores pretenderam expressar. Os resumos em espanhol (resumen) e inglês (abstract) são encaminhados aos revisores de cada idioma.

Finalmente, a versão revisada do manuscrito é encaminhada para diagramação, juntamente com os outros trabalhos que comporão determinado fascículo da revista. Antes da impressão dos exemplares, uma prova gráfica do número é novamente encaminhada ao assistente editorial e ao Conselho Editorial, que realizarão a segunda revisão do fascículo, sob a coordenação do Editor. Finda essa etapa, o artigo poderá enfim vir a lume. Os arquivos são encaminhados para editoração eletrônica da SciELO (Scientific Electronic Library Online), a fim de disponibilizar a versão digital do fascículo, cuja versão impressa será enviada aos autores, corpo editorial, bibliotecas e outros periódicos científicos com os quais a Paidéia estabelece permuta.

\section{Processo editorial - 2007}

No que tange ao processo editorial da Paidéia: Cadernos de Psicologia e Educação no ano de 2007, o tempo médio de tramitação de um manuscrito foi aproximadamente seis meses. A revista recebeu 63 manuscritos, 29 foram aprovados, 14 recusados, sete devolvidos aos autores por não se enquadrarem às normas de publicação, sendo que 13 ainda encontravam-se em processo editorial na ocasião da redação deste relatório. Desse modo, a Paidéia, durante o ano de 2007, aprovou $46 \%$ dos manuscritos submetidos e recusou $22 \%$ deles, conforme evidencia a Tabela 3 .

Tabela 3

Quantidade e situaçäo editorial dos mamuscritos submetidos à revista Paidéia em 2007

\begin{tabular}{lc}
\hline \multicolumn{1}{c}{ Parecer final } & $n$ \\
\hline Aprovado & 29 \\
Recusado & 14 \\
Devolvido & 7 \\
Em processo editorial & 13 \\
Total & 63 \\
\hline
\end{tabular}

Quanto à categoria dos artigos publicados, $45 \%$ constituíram pesquisas teóricas, $48 \%$ empíricas, além de $6 \%$ de resenhas (Tabela 4 ).

Tabela 4

Categoria dos mamuscritos publicados na revista Paidéia no ano de 2007

\begin{tabular}{lcc}
\hline \multirow{2}{*}{ Tipo de trabalho } & \multicolumn{2}{c}{ Período } \\
\cline { 2 - 3 } & \multicolumn{2}{c}{2007} \\
\hline Pesquisas Teóricas & 15 & 45 \\
Pesquisas Empíricas & 16 & 48 \\
Resenha & 2 & 6 \\
Total & 33 & \\
\hline
\end{tabular}

A revista publicou artigos provenientes de 16 universidades/faculdades brasileiras, com predomínio da Universidade de São Paulo e da Universidade de Brasília. Vieram a lume também artigos internacionais provenientes de quatro universidades, a saber: Ankara University, Turquia, Universidade de Coimbra, 
Tabela 5

Origem institucional dos artigos publicados na revista Paidéia no ano de $2007^{*}$

\begin{tabular}{lc}
\hline & Período \\
\cline { 2 - 2 } \multicolumn{1}{c}{ Instituição } & 2007 \\
& $n$ \\
\hline Nacionais & 9 \\
Universidade de São Paulo, SP & 4 \\
Universidade de Brasilia, DF & 3 \\
Universidade Estadual Paulista, SP & 3 \\
Universidade São Francisco, SP & 2 \\
Universidade Federal da Paraiba, PB & 2 \\
Universidade Federal de São João del Rei, MG & 1 \\
Faculdade de Tecnologia e Ciências, BA & 1 \\
Pontifícia Universidade Católica de Campinas, SP & 1 \\
Universidade Estadual de Campinas, SP & 1 \\
Universidade Estadual de Ciências da Saúde de Alagoas, AL & 1 \\
Universidade Federal do Rio Grande do Sul, RS & 1 \\
Universidade Vale do Rio Verde, MG & 1 \\
Universidade Federal de Santa Catarina, SC & 1 \\
Universidade Federal de São Carlos, SP & 1 \\
Universidade Federal do Piauí, SP & 1 \\
Universidade Federal de Uberlândia, MG & \\
\hline Internacionais & 1 \\
Ankara University, Turquia & 1 \\
Universidade do Minho, Portugal & 1 \\
Universidade Fernando Pessoa, Portugal & 1 \\
Universidade de Coimbra, Portugal &
\end{tabular}

Universidade do Minho, Universidade Fernando Pessoa, sendo as três últimas de Portugal (Tabela 5).

No que concerne à procedência dos consultores ad hoc, nota-se o predomínio de revisores lotados em universidades do Estado de São Paulo $(60,9 \%)$, que somados aos avaliadores oriundos de outras instituições pertencentes à região Sudeste, totalizaram $74,4 \%$ do corpo de revisores. A região Sul comportou $12,6 \%$ dos assessores e as regiões Nordeste e Centro-Oeste perfizeram 6,3\% deles cada uma (Tabela 6).

\section{Considerações finais}

O presente relatório visou tornar pública a gestão e tramitação editorial da Paidéia: Cadernos de Psicologia e Educação, o que permitiu descrever seu perfil atual. Os dados apresentados contribuem com subsídios que permitem considerar esse periódico científico em relação às outras publicações da área. Como é do conhecimento dos membros da academia, a editoração de um periódico não é tarefa simples, uma vez que não se limita aos critérios de formalização e conteúdo dos artigos e fascículos, já que questões relacionadas à ética em pesquisa e qualidade de publicação perpassam rotineiramente nossas discussões. Ademais, temos ciência de que os critérios de arbitragem adotados pela revista também gozam de restrições, na medida em que a revisão cega por pares da comunidade científica não é tarefa absolutamente neutra, por mais que a autoria de um manuscrito seja desconhecida, o que redobra as responsabilidades e cuidado da Comissão Editorial dessa revista, sempre zelosa por promover avaliações justas dos trabalhos submetidos. 
Tabela 6

Abrangencia do corpo de consultores ad hoc da revista Paidéia conforme origem geográfica

\begin{tabular}{lcc}
\hline \multicolumn{1}{c}{ Estado } & $\boldsymbol{n}$ & $\mathbf{\%}^{\star}$ \\
\hline São Paulo & 67 & 60,9 \\
Rio de Janeiro & 8 & 7,2 \\
Minas Gerais & 6 & 5,4 \\
Espirito Santo & 1 & 0,9 \\
TOTAL & & \\
SUDESTE & 82 & 74,4 \\
\hline Rio Grande do Sul & 8 & 7,2 \\
Paraná & 6 & 5,4 \\
TOTAL & & \\
SUL & 14 & 12,6 \\
\hline Bahia & 4 & 3,6 \\
Pernambuco & 1 & 0,9 \\
Rio Grande do Norte & 1 & 0,9 \\
Paraiba & 1 & 0,9 \\
TOTAL & & \\
NORDESTE & 7 & 6,3 \\
\hline Brasilia & 5 & 4,5 \\
Goiás & 1 & 0,9 \\
Mato Grosso do Sul & 1 & 0,9 \\
TOTAL & & \\
CENTRO-OESTE & 7 & 6,3 \\
\hline * Cailouros & &
\end{tabular}

* Cálculo baseado no total de 110 assessores.

Quanto às perspectivas de futuro da revista Paidéia: Cadernos de Psicologia e Educação, com sua inserção no sistema SciELO, espera-se continuar fazendo jus ao reconhecimento de sua qualidade como veículo para publicação de artigos originais de pesquisa, tanto do Brasil, quanto da América e da Europa. Mantém-se o compromisso de um trabalho sistemático no sentido de cumprir critérios exigidos pelas diferentes agências que avaliam a qualidade dos veículos de publicação na área das Ciências Humanas no Brasil, e de buscar sempre o aperfeiçoamento e transparência dos critérios de avaliação que norteiam o processo editorial. Cabe salientar que, além dos esforços meritórios empreendidos pelos periódicos científicos da área da Psicologia a fim de atender às diretrizes da base Qualis, acreditamos que cada publicação deva ter qualidades próprias/originais, que decorrem da trajetória profissional/pessoal do corpo editorial que a forjou, o que não impede o atendimento dos critérios supracitados. Além disso, espera-se contar com maior alocação de recursos materiais e humanos, indispensáveis para que possamos realizar as atribuições destinadas às revistas científicas, em especial na área da Psicologia e Educação.

A Paidéia mantém seu compromisso de abertura à diversidade, com o acolhimento de linhas e perspectivas diversificadas, empenhando-se para se consolidar como um veículo de difusão e diálogo do conhecimento nas áreas da Psicologia e da Educação e em suas interfaces com áreas afins.

Diante do aumento da produção científica em escala nacional da qual participamos e assistimos, cabe a permanente reflexão sobre o papel desempenhado pelos periódicos científicos nesse processo. Acreditamos que essa reflexão crítica não pode ser pautada apenas em índices bibliométricos, que tampouco devem ser negligenciados, haja vista que permitem mapear objetivamente os quadros da produção intelectual nacional e internacional. No âmbito da Psicologia é notório o papel destacado da base Qualis, coordenada pela comissão CAPESANPEPP, que estimulou a padronização não apenas dos principais periódicos científicos da área, como também daqueles que estão em processo de consolidação, o que contribuiu significativamente para a formalização dos trabalhos científicos, imprescindível na comunicação entre pares.

Por outro lado, a análise acerca do espaço editorial científico que ocupamos deve pautar-se em sua contextualização histórica e social, ou seja, nos recursos e critérios que o próprio campo do conhecimento utiliza para se reproduzir e avaliar, não nos furtando de pensarmos acerca dos motivos das concessões, contradições e disputas, evidentes ou veladas, que tensionam as relações estabelecidas entre os pesquisadores que engendram a produção intelectual, sob pena de relegarmos nossa função à mera concepção e execução de um trabalho burocrático, para além de nossos relativamente confortáveis gabinetes acadêmicos. 
Endereço para correspondência:

Prof. Dr. Manoel Antônio dos Santos. Universidade de São Paulo. Faculdade de Filosofia, Ciências e Letras de Ribeirão Preto. Departamento de Psicologia e Educação. Av. dos Bandeirantes, 3900. CEP: 14040-901. Ribeirão Preto-SP, Brasil. E-mail: masantos@ffclrp.usp.br

Manoel Antônio dos Santos é Professor Doutor da Faculdade de Filosofia, Ciências e Letras de Ribeirão Preto da Universidade São Paulo, Editor Responsável pela revista Paidéia: Cadernos de Psicologia e Educação.

Lucy Leal Melo-Silva é Professor Doutor da Faculdade de Filosofia, Ciências e Letras de Ribeirão Preto da Universidade de São Paulo, Editora Associada da revista Paidéia: Cadernos de Psicologia e Educação.

Eduardo Name Risk é psicólogo e bacharel em Psicologia pela Faculdade de Filosofia, Ciências e Letras de Ribeirão Preto da Universidade de São Paulo, Assistente editorial da revista Paidéia: Cadernos de Psicologia e Educação. 